\title{
Etiological Profile of Cirrhosis in a Tertiary Care Institute in Northern India
}

${ }^{1}$ Prerna Goyal, ${ }^{2}$ Omesh Goyal, ${ }^{3}$ Deepinder Kaur, ${ }^{4}$ Rajoo S Chhina

\begin{abstract}
Introduction: Cirrhosis of the liver is associated with increased morbidity and mortality. There is paucity of data on the etiological profile of cirrhosis in Punjab. The purpose of this study was to evaluate the etiological profile of liver cirrhosis in adults in a tertiary care institute in northern India.
\end{abstract}

Materials and methods: This study included all adult patients with cirrhosis admitted to the Gastroenterology unit of a tertiary care institute in northern India from January 2013 to December 2016. The diagnosis of cirrhosis was made based on the clinical, radiological, biochemical, and/or histological criteria. Patients with incomplete etiological workup were excluded.

Results: A total of 716 hospitalized patients with cirrhosis were included. The mean age of patients was $54 \pm 9.3$ years (18-82 years), and male:female ratio was 5.7:1. Most common etiologies of cirrhosis were: alcohol $(49.2 \% ; n=352)$, hepatitis C virus (HCV) infection (29.4\%; 211), and nonalcoholic fatty liver disease (NAFLD) (13.6\%; 98). Hepatitis B virus (HBV) infection was identified in $3.9 \%(n=28)$ patients only. Other uncommon causes of cirrhosis were: autoimmune $(n=8$; $1.1 \%)$, Wilson's disease $(n=5 ; 0.7 \%)$, celiac disease $(n=$ 3; $0.4 \%)$, Budd-Chiari syndrome (BCS) $(n=1 ; 0.1 \%)$, and cryptogenic ( $n=10 ; 1.4 \%)$.

Conclusion: In this study, alcohol and HCV infection were the most common causes of cirrhosis. Preventive measures are urgently required to control these factors to decrease morbidity and mortality.

Keywords: Cirrhosis, Hepatitis, Prevalence.

How to cite this article: Goyal P, Goyal O, Kaur D, Chhina RS. Etiological Profile of Cirrhosis in a Tertiary Care Institute in Northern India. J Gastrointest Infect 2018;8(1):28-31.

Source of support: Nil

Conflict of interest: None

\footnotetext{
${ }^{1}$ Former Consultant, ${ }^{2}$ Associate Professor, ${ }^{3}$ Professor and Head ${ }^{4}$ Dean Academics and Professor

${ }^{1}$ Department of Medicine, Dayanand Medical College and Hospital Ludhiana, Punjab, India

${ }^{2,4}$ Department of Gastroenterology, Dayanand Medical College and Hospital, Ludhiana, Punjab, India

${ }^{3}$ Department of Microbiology, Dayanand Medical College and Hospital, Ludhiana, Punjab, India
}

Corresponding Author: Omesh Goyal, Associate Professor Department of Gastroenterology, Dayanand Medical College and Hospital, Ludhiana, Punjab, India, e-mail: goyalomesh@yahoo. co.in

\section{INTRODUCTION}

Liver cirrhosis is associated with both direct complications of the loss of liver function and portal hypertension, such as jaundice, encephalopathy, or variceal hemorrhage and indirect complications like hepatorenal syndrome, hepatocellular carcinoma, or infections. ${ }^{1}$ These complications of cirrhosis lead to high morbidity and mortality, thus making this disease a major cause of global health burden. According to the Global Burden of Disease 2010 study, liver cirrhosis was the cause of $1.2 \%$ of the global Disability-Adjusted Life Years, and $2 \%$ of all deaths worldwide in year 2010., ${ }^{2,3}$ In India, since 1980, there has been a gradual increase in mortality due to cirrhosis, as the prevalence of risk factors of cirrhosis, i.e., alcohol intake, $\mathrm{HBV}, \mathrm{HCV}$, and diabetes (a major risk factor for NAFLD), is also increasing. ${ }^{4}$ In the year 2010, deaths due to liver cirrhosis in India accounted for almost one-fifth $(18.3 \%)$ of the liver cirrhosis deaths worldwide. ${ }^{5}$ Therefore, universal and stringent efforts are required to control the preventable risk factors for cirrhosis.

The risk factors for cirrhosis vary among different geographical populations worldwide. In developed countries, alcohol and HCV infection are major causes of chronic liver disease, while in developing countries, alcohol and HBV infection are the major etiological causes. A systematic review by $\mathrm{D}^{\prime} \mathrm{Amico}$ et $\mathrm{al}^{6}$ including 118 studies (23,797 patients) reported alcohol (46\%) as the most common cause of cirrhosis, followed by HCV $(35 \%), \mathrm{HBV}(11 \%)$, and other causes $(12 \%)$.

In India as well, the risk factors of cirrhosis have varied among various studies conducted in different geographical areas. Data on the etiological aspects of cirrhosis of the liver from Punjab, a northern Indian state, are insufficient. This study aimed to evaluate the etiological profile of patients with cirrhosis admitted to a tertiary care institute in Punjab, India.

\section{MATERIALS AND METHODS}

This study included all adult patients with cirrhosis admitted to the gastroenterology unit of a tertiary care hospital in northern India from January 2013 to December 2016. The diagnosis of cirrhosis was made based on clinical, radiological, biochemical, and/or histological criteria. Clinical history, examination, and laboratory 
details including hemogram, liver and renal function tests, prothrombin time index, fasting blood sugar, and $\alpha$-feto protein were noted. Etiology of cirrhosis was considered to be alcohol if the alcohol consumption was $>60$ to $80 \mathrm{gm} /$ day for men and $>20$ to $40 \mathrm{gm} /$ day for women for more than 10 years. Markers of viruses, such as hepatitis B surface antigen and anti-HCV antibody were done in each patient. Serological markers like antinuclear antibodies, antibody against liver/kidney microsomes, antismooth muscle antibodies, and immunoglobulin A tissue transglutaminase antibody were done in patients who were nonalcoholic and negative for viral markers. Investigations for Wilson's disease (serum ceruloplasmin, 24-hour urinary copper and slit lamp examination for Kayser-Fleischer ring), hemochromatosis, and BCS (venous Doppler, etc.) were done where indicated. The NAFLD was considered as the possible cause for cirrhosis in all obese patients, in whom no other etiological factor of cirrhosis was present. Obesity was defined by Asian criteria (body mass index $>27.5$ ) considering lifetime highest weight. The etiology was labeled cryptogenic when no cause of cirrhosis could be identified. Computed tomography was done if there was a suspicion of hepatocellular carcinoma on ultrasound. Upper gastrointestinal endoscopy was performed in all patients unless contraindicated or refused by patient. Exclusion criteria were: patients with incomplete etiological workup and age $<18$ years. Data are presented as mean \pm standard deviation for quantitative variables, and proportions for qualitative variables. A $p$ value $<0.05$ was considered statistically significant.

\section{RESULTS}

A total of 716 hospitalized patients with cirrhosis were included. The mean age of patients was $54 \pm 9.3$ years, with a range from 18 to 82 years. The male:female ratio was 5.7:1 (609/107). The various etiological factors of cirrhosis are shown in Graph 1.

The frequency distribution of etiology of cirrhosis according to age and gender is shown in Tables 1 and 2 .

Overall, alcohol was the most common cause of cirrhosis. Alcohol intake was reported in all age groups with maximum prevalence in age group of 40 to 49 years (35.5\%). Alcohol was the commonest cause of cirrhosis in males $(57.8 \%)$, while HCV was the commonest cause of cirrhosis among females (68.2\%). Overall, of 211 patients with HCV infection, 68 patients had history of significant alcohol intake, and 22 were obese. The mean amount of alcohol consumption among males was $210 \mathrm{gm} /$ day, while none of the female patient had history of significant alcohol intake. The mean duration of alcohol intake was 18 years (12-35 years). The HBV infection was identified in $3.9 \%(n=28)$ patients only, of which two patients had

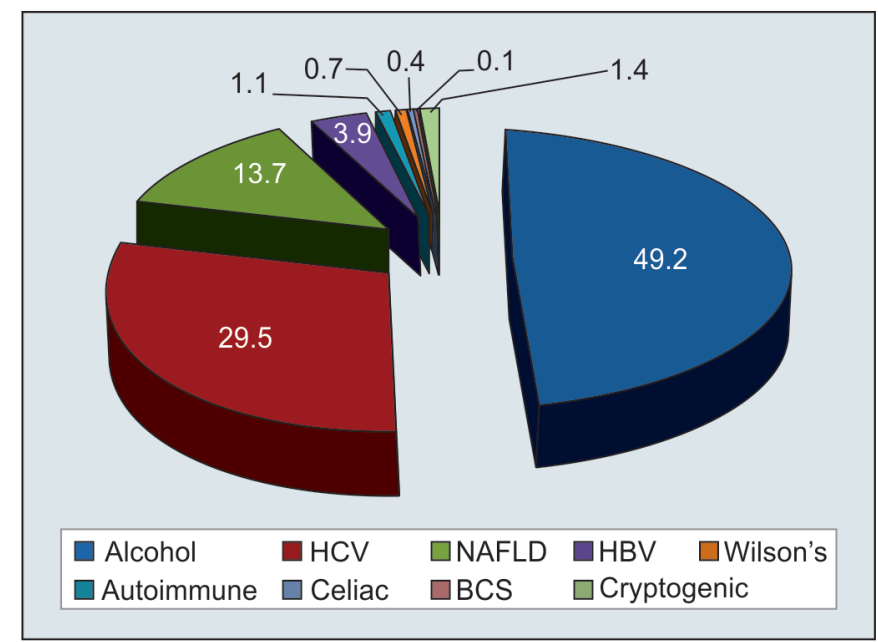

Graph 1: Etiological factors for liver cirrhosis

Table 1: Frequency distribution of etiology of cirrhosis according to age

\begin{tabular}{llllllllll}
\hline Age (years) & Alcohol & HCV & NAFLD & HBV & Autoimmune & $\begin{array}{l}\text { Wilson's } \\
\text { disease }\end{array}$ & $\begin{array}{l}\text { Celiac } \\
\text { disease }\end{array}$ & $\begin{array}{l}\text { Cryptogenic } \\
\text { etiology }\end{array}$ \\
\hline $18-29$ & 3 & 2 & 0 & 0 & 0 & 2 & 0 & 0 & 1 \\
$30-39$ & 78 & 36 & 8 & 0 & 3 & 2 & 0 & 0 & 2 \\
$40-49$ & 125 & 81 & 31 & 13 & 2 & 1 & 1 & 1 & 0 \\
$50-59$ & 96 & 71 & 42 & 9 & 3 & 0 & 2 & 0 & 4 \\
$60-69$ & 42 & 17 & 15 & 4 & 0 & 0 & 0 & 0 & 1 \\
$>70$ & 8 & 4 & 2 & 2 & 0 & 0 & 0 & 0 & 2 \\
Total & 352 & 211 & 98 & 28 & 8 & 5 & 3 & 1 & 10 \\
\hline
\end{tabular}

Table 2: Frequency distribution of etiology of cirrhosis according to gender

\begin{tabular}{llllllllll}
\hline Gender & Alcohol & HCV & NAFLD & HBV & Autoimmune & $\begin{array}{l}\text { Wilson's } \\
\text { disease }\end{array}$ & $\begin{array}{l}\text { Celiac } \\
\text { disease }\end{array}$ & $\begin{array}{l}\text { Cryptogenic } \\
\text { etiology }\end{array}$ \\
\hline Male & 352 & 138 & 82 & 22 & 1 & 4 & 1 & 0 & 8 \\
Female & 0 & 73 & 16 & 6 & 7 & 1 & 2 & 1 & 2 \\
\hline Total & 352 & 211 & 98 & 28 & 8 & 5 & 3 & 1 & 10 \\
\hline
\end{tabular}


history of significant alcohol intake. None of the patients had combined HBV and HCV infection. The NAFLD was diagnosed in 98 patients, while other causes of cirrhosis included autoimmune etiology in 8 patients $(1.1 \%)$, Wilson's disease in 5 patients $(0.7 \%)$, celiac disease in $3(0.4 \%)$, and BCS in $1(0.1 \%)$. In 10 patients $(1.4 \%)$, no etiology could be identified and were labeled as cryptogenic etiology.

\section{DISCUSSION}

Cirrhosis is progressive and chronic scarring of the liver caused by persistent injury. Various etiological factors of cirrhosis include alcohol, HBV, HCV, NAFLD, autoimmune, biliary cirrhosis, cardiac cirrhosis, celiac disease, inherited metabolic liver diseases, including hemochromatosis, Wilson's disease, $\alpha 1$ antitrypsin deficiency, etc. Liver cirrhosis is labeled as cryptogenic if no underlying cause can been identified. There has been a wide variation in the etiological factors of cirrhosis worldwide. Alcohol has been reported as the commonest etiological factor for cirrhosis in countries with high alcohol consumption, whereas in countries with low alcohol consumption, viral hepatitis is the most common cause of cirrhosis. ${ }^{7}$

Among most of the Indian studies from various regions, HBV has been reported as the leading cause of cirrhosis. A study by Sarin et $\mathrm{al}^{8}$ reported HBV as the commonest cause of cirrhosis in adults (44.3\%) as well as the young $(50.8 \%)$, followed by alcohol in both adults (35.8\%) and the young (15.9\%). A study from eastern India including 175 patients with chronic liver disease reported $\mathrm{HBV}(35.4 \%), \mathrm{HCV}(14.9 \%)$, autoimmunity $(2.8 \%)$, Wilson's disease $(2.8 \%)$, and alcohol $(1.7 \%)$ as the commonest etiological factors of cirrhosis. No etiological factor could be identified in $15.8 \%$ patients. ${ }^{9}$ A study by Trimukhe et $\mathrm{al}^{10}$ from Madhya Pradesh reported HBV $(40.6 \%)$ as the leading cause for cirrhosis followed by alcohol (33.3\%), hepatitis C (3.3\%), nonalcoholic steatohepatitis (NASH) $(2.2 \%)$, and cryptogenic $(19.4 \%)$. In a recent study from Himachal Pradesh, alcohol was the leading cause $(62.9 \%)$, followed by HBV (10.1\%), NASH (7.9\%), autoimmune (3.9\%), HCV (2.8\%), Wilson disease, cardiac cirrhosis and cryptogenic $(9.6 \%) .{ }^{11} \mathrm{~A}$ recent multicentric study reported significant interregional differences in the etiology of chronic liver disease (hepatitis B in East and South, hepatitis C in North, alcohol in Northeast, NAFLD in West). Alcoholism (34.3\% of 4413) was the commonest cause of cirrhosis, while HBV (33. 3\%) was the major cause of chronic liver disease in general and noncirrhotic chronic liver disease $(40.8 \%$ out of 8163). ${ }^{12}$ In the present study, alcohol was the most common cause of cirrhosis, followed by HCV, NAFLD, HBV, and autoimmune.
Alcohol consumption has been one of the major causes of liver cirrhosis among most of the studies from India. As reported by the World Health Organization, in population $>15$ years of age, the per capita alcohol consumption (in liters of pure alcohol) in India in the year 2010 was 4.3. In the year 2012, the age-standardized death rates due to liver cirrhosis in India among males and females in year 2012 were 39.5 and 19.6 respectively, of which alcohol attributable fractions constituted 62.9 and $33.2 \%$ respectively. ${ }^{13}$ A recent epidemiological survey was conducted by the Department of Psychiatry, Govt. Medical College and Hospital, Chandigarh, to estimate the pattern of alcohol and other substance dependence in rural and slum dweller population of Chandigarh. ${ }^{14}$ In this survey, out of the total population of 2,992 subjects surveyed, $6.88 \%$ of individuals fulfilled dependence criteria of International Classification of Diseases-10. Both among urban slum and rural areas, alcohol was the primary substance of dependence. Age at first drug use was $19.75 \pm 5.4$ years in urban slums and $20.89 \pm 5.31$ years among rural population. ${ }^{14}$

The HCV infection is one of the major causes of parentally acquired hepatitis worldwide. An estimated 200 million people are infected with $\mathrm{HCV}$ infection worldwide. Chronic HCV infection is associated with a significant risk of development of cirrhosis and hepatocellular carcinoma. ${ }^{15}$ The HCV infection was one of the major causes of cirrhosis in our study, similar to the worldwide data, but it is in contrast to the data from other Indian studies. This is possibly due to the high prevalence of $\mathrm{HCV}$ infection in general population in Punjab, as compared with other parts of the country. A study by Sood et $\mathrm{al}^{16}$ reported 5.2\% prevalence of anti-HCV antibodies in a selected geographical region of Punjab. Another study done in Kolkata showed the prevalence of HCV infection among patients to be $1.5 \%$, whereas studies done in Delhi showed the prevalence of HCV infection to be 5.5\%. ${ }^{17,18}$

The NAFLD was the third most common etiological factor for cirrhosis in the present study. The prevalence of NAFLD in India is gradually increasing due to the rise in prevalence of metabolic risk factors including diabetes mellitus, obesity, dyslipidemia, etc. Patients who have NAFLD have been shown to be at increased risk for liver-related morbidity and mortality. In addition, these patients also have increased incidence of diabetes mellitus and increased cardiovascular disease risk on long-term follow-up. ${ }^{19}$

The HBV as an etiological factor for cirrhosis was less frequent in our study. This is likely due to the low prevalence of $\mathrm{HBV}(1.3 \%)$ in the general population in our region as compared with the rest of India. ${ }^{16}$ The average estimated carrier rate of HBV in India is $4 \%$, with a total pool of approximately 36 million carriers. ${ }^{20}$ 
Liver cirrhosis-related mortality accounts for a substantial disease burden worldwide. Over the past 30 years, cirrhosis-related mortality has increased steadily, exceeding one million in 2010 , or approximately $2 \%$ of all deaths in that year. ${ }^{5}$ In India, liver cirrhosis-related mortality has increased to 188,575 (95\% uncertainty levels $109,748-303,989)$ in 2010 from 77,741 (95\% uncertainty levels $52,196-116,746)$ in $1980 .^{5}$

Treatment of chronic liver disease is expensive and requires long-term follow-up. Once cirrhosis develops, liver transplant is the only possible cure in most of the cases. In patients with decompensated liver cirrhosis, symptomatic treatment provides minimal survival benefit. Therefore, identifying and controlling the risk factors for cirrhosis are the need of the hour. Steps to improve prevention and treatment of risk factors of liver cirrhosis will decrease the disease burden.

The favorable points of this study are that it reports the various risk factors for cirrhosis in Punjab, the data on which were limited. The identification of risk factors will help to target preventive measures and make appropriate treatment decisions. Focus on primary preventive measures like abstinence from alcohol, prevention of $\mathrm{HCV}$ and HBV transmission, hepatitis B vaccination, and management of metabolic syndrome by diet, healthy lifestyle, and drugs are required.

\section{REFERENCES}

1. Thulstrup AM, Sorensen HT, Schonheyder HC, Moller JK, Tage-Jensen U. Population-based study of the risk and shortterm prognosis for bacteremia in patients with liver cirrhosis. Clin Infect Dis 2000 Dec;31(6):1357-1361.

2. Murray CJ, Vos T, Lozano R, Naghavi M, Flaxman AD, Michaud C, Ezzati M, Shibuya K, Salomon JA, Abdalla S, et al. Disability-adjusted life years (DALYs) for 291 diseases and injuries in 21 regions, 1990-2010: a systematic analysis for the Global Burden of Disease Study 2010. Lancet 2012 Dec;380(9859):2197-2223.

3. Lozano R, Naghavi M, Foreman K, LimS, Shibuya K, Aboyans V, Abraham J, Adair T, Aggarwal R, Ahn SY, et al. Global and regional mortality from 235 causes of death for 20 age groups in 1990 and 2010: a systematic analysis for the Global Burden of Disease Study 2010. Lancet 2012 Dec;380(9859): 2095-2128.

4. Tanaka H, Tsukuma H, Yamano H, Oshima A, Shibata H. Prospective study on the risk of hepatocellular carcinoma among hepatitis $\mathrm{C}$ virus-positive blood donors focusing on demographic factors, alanine aminotransferase level at donation and interaction with hepatitis B virus. Int J Cancer 2004 Dec;112(6):1075-1080.

5. Mokdad AA, Lopez AD, Shahraz S, Lozano R, Mokdad AH, Stanaway J, Murray CJ, Naghavi M. Liver cirrhosis mortality in 187 countries between 1980 and 2010: a systematic analysis. BMC Med 2014 Sep;12:145.

6. D'Amico G, Garcia-Tsao G, Pagliaro L. Natural history and prognostic indicators of survival in cirrhosis: a systemic review of 118 studies. J Hepatol 2006 Jan;44(1):217-231.

7. Schuppan D, Afdhal NH. Liver cirrhosis. Lancet 2008 Mar; 371(9615):838-851.

8. Sarin SK, Chari S, Sundaram KR, Ahuja RK, Anand BS, Broor SL. Young $v$ adult cirrhotics: a prospective, comparative analysis of the clinical profile, natural course and survival. Gut 1988 Jan;29(1):101-107.

9. Ray G, Ghoshal UC, Banerjee PK, Pal BB, Dhar K, Pal AK, Biswas PK. Aetiological spectrum of chronic liver disease in eastern India. Trop Gastroenterol 2000 Apr-Jun;21(2):60-62.

10. Trimukhe R, Rai R, Narayankar SM, Shewale S, Jagtap N, Rai S, Parashar MK. Epidemiological spectrum of chronic liver disease in eastern Madhya Pradesh India. J Assoc Physicians India 2011 Jan;59:48.

11. Sharma B,MarwahR, RainaS,Sharma N,KaushikM,KaushalSS. A study on the etiology of cirrhosis of liver in adults living in the Hills of Himachal Pradesh, India. Trop Gastroenterol 2016 Jan;37(1):37-41

12. Mukherjee PS, Vishnubhatla S, Amarapurkar DN, Das K, Sood A, Chawla YK, Eapen CE, Boddu P, Thomas V, Varshney $\mathrm{S}$, et al. Etiology and mode of presentation of chronic liver diseases in India: a multi centric study. PLoS One 2017 Oct;12(10):e0187033.

13. WHO. Global status report on alcohol and health 2014. Geneva: WHO; 2014. Available from: www.who.int/substance_abuse/publications/global_alcohol_report/en/.

14. Chavan BS, Arun P, Bhargava R, Singh GP. Prevalence of alcohol and drug dependence in rural and slum population of Chandigarh: a community survey. Indian J Psychiatry 2007 Jan;49(1):44-48.

15. European Association for the Study of the Liver. EASL recommendations on treatment of hepatitis C 2016. J Hepatol 2016 Jan;66(1):153-194.

16. Sood A, Sarin SK, Midha V, Hissar S, Sood N, Bansal P, Bansal M. Prevalence of hepatitis $C$ virus in a selected geographical area of northern India: a population based survey. Indian J Gastroenterol 2012 Sep;31(5):232-236.

17. Chakraborty A, PramanikSB, Roy DS, SarkarS, Chakraborty M, Nandi A. A retrospective study on the sero-prevalence of hepatitis $C$ infection in a tertiary care hospital in Kolkata, India. Int J Curr Microbiol App Sci 2015;4(3):115-123.

18. Rajani M, Jais M. Age-wise seroprevalence of hepatitis $C$ virus infection in clinically suspected infectious hepatitis patients attending a tertiary care hospital in Delhi. Int J Med Pub Health 2014 Jan-Mar;4(1):78-81.

19. Duseja A, Singh SP, Saraswat VA, Acharya SK, Chawla YK, Chowdhury S, Dhiman RK, Jayakumar RV, Madan K, Misra SP, et al. Non-alcoholic fatty liver disease and metabolic syndrome-position paper of the Indian National Association for the Study of the Liver, Endocrine Society of India, Indian College of Cardiology and Indian Society of Gastroenterology. J Clin Exp Hepatol 2015 Mar;5(1):51-68.

20. Tandon BN, Acharya SK, Tandon A. Epidemiology of hepatitis B virus infection in India. Gut 1996;38(Suppl 2):S56-S59. 